baseline levels of activated caspase-1, which cannot be significantly increased after stimulation. Altogether, our data supports the hypothesis that caspase-1 is activated in early arthritis.

\section{A4 CASPASE-1 IS ACTIVE SINCE THE EARLY PHASE OF RHEUMATOID ARTHRITIS}

Rita Cascão, Joaquim Polido-Pereira, ${ }^{1,2}$ Helena Canhão, ${ }^{1,2}$ Ana Maria Rodrigues, ${ }^{1,2}$ Márcio Navalho, ${ }^{1,3}$ Helena Raquel, ${ }^{1}$ Ana Filipa Mourão, ${ }^{1,4}$ Catarina Resende, ${ }^{2}$ José Alberto Pereira da Silva, ${ }^{2}$ João Eurico Fonseca, ${ }^{1,2}$ Luis Ferreira Moita ${ }^{1} 1 / n s t i t u t o$ de Medicina Molecular, Faculdade de Medicina da Universidade de Lisboa, Lisbon, Portugal; ${ }^{2}$ Rheumatology Department, Centro Hospitalar de Lisboa Norte, EPE, Hospital de Santa Maria, Lisbon, Portugal; ${ }^{3}$ Radiology Department, Hospital da Luz, Lisbon, Portugal; ${ }^{4}$ Rheumatology Department, Centro Hospitalar de Lisboa Ocidental, EPE, Hospital Egas Moniz, Lisbon, Portugal

JEF and LFM are joint senior authors.

10.1136/ard.2010.149096.4

Background We have previously reported an increase in interleukin (IL)-1 $\beta$ levels in very recent onset arthritis. Therefore, molecular mechanisms that regulate IL-1 $\beta$ secretion, such as caspase- 1 activation, might play a relevant role in arthritis onset and progression.

Objectives Our main goal was to evaluate caspase-1 state of activation in early polyarthritis and established rheumatoid arthritis (RA) patients.

Materials and methods Blood samples were collected from 12 untreated early polyarthritis patients, 12 established RA patients and 10 healthy controls. Caspase- 1 activity was assessed using a carboxyfluorescein FLICA detection kit.

Results We found that both early polyarthritis and established RA patients have higher active caspase-1 levels. Surprisingly, caspase-1 activation levels in patient cells remain unchanged when cells were stimulated with Escherichia coli and ATP, whereas in control cells caspase-1 levels increased after stimulation. We observed no significant differences when comparing early polyarthritis with established RA samples. However, in RA patients with higher disease activity the proportion of active caspase- 1 is higher.

Conclusions Our results suggest that leucocytes in both early polyarthritis and established RA patients have high 